\title{
Relação entre ansiedade e modulação autonômica cardíaca
}

\author{
Relationship between anxiety and autonomic heart modulation
}

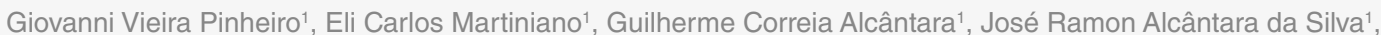 \\ Maria do Socorro da Silva ${ }^{1}$, Larissa Raylane Lucas Monteiro ${ }^{1}$, Luana Bezerra Mangueira' ${ }^{1}$, Yasmim Mota de Moraes ${ }^{1}$, \\ Vitor Engrácia Valenti², Milana Drumond Ramos Santana ${ }^{1}$
}

${ }^{1}$ Núcleo de Estudos em Ciências Fisiológicas e da Coletividade, Faculdade de Juazeiro do Norte(FNJ) - Juazeiro do Norte (CE), Brasil.

${ }^{2}$ Centro de Estudos do Sistema Nervoso Autônomo (CESNA), Departamento de Fonoaudiologia, Faculdade de Filosofia e Ciências, (UNESP) - Marília (SP), Brasil.

DOI: http://dx.doi.org/10.7322/abcshs.v43i3.1092

\section{RESUMO}

A ansiedade é caracterizada pelo sentimento de tensão, nervosismo, apreensão e preocupação que pode alterar a modulação do sistema nervoso autônomo sobre a condução elétrica ligada ao coração, determinando a intensidade nos batimentos cardíacos modificando a variabilidade da frequência cardíaca que é um importante indicador de condições normais e patológicas. Assim, o objetivo do estudo foi analisar a relação existente entre a ansiedade e o processo de regulação autonômica cardíaca. Este estudo trata-se de uma revisão integrativa desenvolvido por meio de buscas nas bases de dados da Lilacs, Medline e SciELO, com os descritores em ciências da saúde e operador booleano: ansiedade AND frequência cardíaca e, ansiedade AND sistema nervoso autônomo. A busca foi realizada durante o mês de setembro de 2017, sendo incluídos artigos disponíveis na íntegra; em português e inglês e; publicados nos últimos 06 anos. Excluiu-se artigos que não fossem originais, teses, dissertações e monografias. Obteve-se um total de 10 artigos, nos quais observou-se que a ansiedade altera a resposta autonômica, assim quando o corpo entra em tensão, aumentase a frequência cardíaca, o predomínio do sistema simpático e no relaxamento ocorre o domínio parassimpático. Assim indivíduos portadores de ansiedade apresentam menor variação na frequência cardíaca e possuem uma capacidade menor de interação social. Com isto, evidencia-se uma baixa adaptação do sistema nervoso autônomo em casos de ansiedade, sendo que a variabilidade da frequência cardíaca encontra-se reduzida; condição esta que pode ser classificada como patológica.

Palavras-chave: frequência cardíaca; sistema nervoso autônomo; ansiedade.

\begin{abstract}
The anxiety is characterized by the sensation of tension, nervousness, apprehension and worry that can alter the modulation of autonomic nervous system on the electrical conduction linked to the heart, determining the intensity in the heart beats modifying the heart rate variability, which is an important indicator of conditions normal and pathological. Thus, the study objective is to analyze the relationship between anxiety and the process of autonomic cardiac regulation. This study is an integrative review developed through searches in the databases of Lilacs, Medline and SciELO, with the descriptors in health sciences and boolean operator: anxiety AND heart rate and anxiety AND autonomic nervous system. The search was performed during the month of September of 2017, being included articles available in full; in Portuguese and English and; published in the last 6 years. Was excluded articles that were not original, theses, dissertations and monographs. Was obtained a total of 10 articles, in which it was observed that anxiety alters the autonomic response, so when the body is in tension, increases your heart rate, the predominance of the sympathetic system and in the relaxation occurs the domain of the parasympathetic. Thus, individuals with anxiety present lower variation in heart rate and have a lower capacity for social interaction. With this, it is evident a low adaptation of the autonomic nervous system in cases of anxiety, being that the variability of the heart rate is reduced; condition that can be classified as pathological.
\end{abstract}

Keywords: heart rate; autonomic nervous system; anxiety. 


\section{INTRODUÇÃO}

A ansiedade é caracterizada por um mal-estar físico e psicológico que altera as características comportamentais e neurológicas dos indivíduos. Essa condição é provocada pelo desenvolvimento de experiências, medos, traumas e pelo estresse. Sendo manifestada de forma aguda ou crônica ${ }^{1,2}$. Em aspectos de influência, está relacionada com vivências anteriores que foram tidas como, traumatizantes, estressantes, dolorosas ou que de alguma forma não foram positivas ${ }^{2}$.

As manifestações mais comuns da ansiedade são na forma de excesso de planejamento de situações futuras, que induzem uma expectativa sobre o que virá. Outro fator relevante é quando o indivíduo se coloca em situações similares às experiências passadas de traumas. Essas situações desencadeiam uma série de manifestações comportamentais, que tem influência no âmbito social do mesmo. Em graus mais elevados de transtorno de ansiedade, alguns indivíduos podem ter complicações com as atividades sociais, gerando um quadro de isolamento e impossibilidade de realizar atividades diárias, consequentemente, ocasionando impacto negativo na qualidade de vida do ser, e se tornando um empecilho para o desenvolvimento cognitivo, social e emocional ${ }^{2}$.

Tendo em vista as alterações sentimentais causadas pela ansiedade, como a tensão aumentada, o nervosismo e a preocupação, o sistema nervoso autônomo (SNA) sofre ativação podendo ocorrer uma mudança nos impulsos nervosos sobre os movimentos cardíacos ${ }^{1}$.

Nesse contexto, a variabilidade da frequência cardíaca (VFC) é apontada como um indicador para o rastreamento de algumas condições normais e patológicas. Assim, os índices de VFC buscam compreender as oscilações entre os intervalos R-R dos batimentos cardíacos, que são resultado da forma atuante do SNA sobre a frequência cardíaca (FC). Essa atuação exerce controle sobre o sistema cardiovascular através das transmissões pelos nervos ligados ao coração, sendo utilizadas as informações enviadas pelos barorreceptores, quimiorreceptores, receptores atriais e ventriculares e, a partir delas, processa e desencadeia modificações em vários sistemas de compensação: mudanças no sistema respiratório, sistema vasomotor, sistema renina-angiotensina-aldosterona e sistema termorregulador ${ }^{3,4}$.

A FC está ligada, junto às atividades barorreceptoras, a esse controle neuronal. O domínio do sistema nervoso simpático está relacionado ao aumento da $\mathrm{FC}$, enquanto o parassimpático com a diminuição dessa frequência. A VFC relata as variações existentes entre os intervalos dos batimentos cardíacos consecutivos (intervalos R-R). Esses batimentos estão relacionados à influência que o SNA tem sobre o controle do nó sinusal ${ }^{3,5}$.

Desta forma, diante do contexto apresentado, tem-se a seguinte questão: Qual a relação entre VFC e a ansiedade?

Analisar a VFC pode se tornar um método promissor para correlacionar a ansiedade em estudos que buscam o diagnóstico e tratamento dessa enfermidade. Visto que, a VFC é um método não invasivo e popularmente o diagnóstico deste transtorno se dá de uma forma verbal, com base no que o indivíduo com esta condição responde. Assim, a VFC pode ser um método de diagnóstico em indivíduos com distúrbio de ansiedade. Estes podem apresentar índices de VFC diferentes do que de um indivíduo sem esta condição. Assim, a ansiedade pode despertar um estado de alerta e possivelmente gerar uma menor adaptação da modulação autonômica em relação a indivíduos normais, e possivelmente uma maior predominância da condução simpática.

Diante disso, este estudo possuiu como objetivo analisar a relação existente entre a ansiedade e o processo de regulação autonômica cardíaca.

\section{MÉTODO}

O presente estudo trata-se de uma revisão integrativa de literatura. O desenvolvimento deste ocorreu através de buscas nas bases de dados Literatura Latino-Americana e do Caribe em Ciências da Saúde (LILACS), Medical Literature Analysis and Retrieval Sistem online (MEDLINE) via Biblioteca Virtual em Saúde (BVS) e Scientific Electronic Library Online (SCIELO), sendo empregados os Descritores em Ciências da Saúde (DECS) em associação com o operador booleano AND em duas pesquisas distintas: ansiedade AND frequência cardíaca e, ansiedade AND sistema nervoso autônomo.

A busca foi realizada durante o mês de setembro do ano 2017, sendo executada a triagem dos trabalhos, avaliando-os para inclusão: estar disponível na íntegra; estudos em português e inglês; que tivessem sido publicados nos últimos 06 anos. O critério de exclusão adotado foi artigos que não fossem originais, teses, dissertações e monografias.

Foram considerados os índices lineares no domínio do tempo (SDNN: Desvio padrão de todos os intervalos R-R normais gravados em um intervalo de tempo, expresso em ms; RMSSD: Raiz quadrada da média do quadrado das diferenças entre intervalos $\mathrm{R}-\mathrm{R}$ normais adjacentes, em um intervalo de tempo, expresso em ms) e da frequência (HF: Componente de alta frequência com variação de 0,15 a 0,4 Hz; LF: Componente de baixa frequência com variação entre 0,04 e 0,15 Hz; LF/HF: Relação entre os componentes de baixa e alta frequência) e da frequência da VFC 3 .

\section{RESULTADOS}

O presente estudo foi construído com base em 10 artigos que foram selecionados conforme critérios estabelecidos no método, onde, na primeira busca nas bases de dados, utilizando os descritores ansiedade AND frequência cardíaca, foram encontrados um total de 277 estudos, após análise dos trabalhos pelos critérios estabelecidos foram selecionados 06 artigos para a amostra final. Da mesma forma ocorreu a segunda busca, utilizando os descritores ansiedade AND sistema nervoso autônomo, encontrando-se inicialmente 20 estudos e após critérios permaneceram 04 artigos.

A Tabela 1 apresenta um resumo geral dos artigos incluídos na amostra final, estando presentes o nome dos autores, os índices utilizados para mensurar a VFC, os objetivos, as amostras e as principais conclusões, conforme a seguir:

A Tabela 2 também apresenta aspectos citados na anterior, conforme ilustrado a seguir: 
Tabela 1: Síntese dos artigos selecionados para a revisão integrativa na primeira busca

\begin{tabular}{|c|c|c|c|c|}
\hline Autor e ano & $\begin{array}{l}\text { Índices } \\
\text { analisados }\end{array}$ & Objetivos & Amostra & Principais conclusões \\
\hline $\begin{array}{l}\text { Stratford et al. } \\
2014^{8}\end{array}$ & LF; HF; LF/HF & $\begin{array}{l}\text { Investigar a ligação entre a VFC } \\
\text { e a formação de uma aliança } \\
\text { terapêutica (AT). }\end{array}$ & $\begin{array}{l}30 \text { clientes com sintomas } \\
\text { ansiosos ( } 15 \text { homens e } \\
15 \text { mulheres). }\end{array}$ & $\begin{array}{l}\text { Há uma ligação entre AT, ansiedade e VFC. } \\
\text { Uma resposta maior de ansiedade durante } \\
\text { situações de estresse aumentando a atividade } \\
\text { simpática predispõe os indivíduos a serem } \\
\text { mais vulneráveis à doenças cardíacas. }\end{array}$ \\
\hline $\begin{array}{l}\text { Wells et al. } \\
2012^{11}\end{array}$ & HF; LF/HF & $\begin{array}{l}\text { Explorar a eficácia do } \\
\text { biofeedback como uma } \\
\text { intervenção para a ansiedade } \\
\text { de desempenho musical. }\end{array}$ & $\begin{array}{l}45 \text { músicos, sendo } 24 \\
\text { mulheres e } 21 \text { homens, } \\
\text { com idade entre } 19 \text { e } \\
67 \text { anos. }\end{array}$ & $\begin{array}{l}\text { A respiração lenta independente do } \\
\text { biofeedback é capaz de controlar a excitação } \\
\text { fisiológica em antecipação ao estresse } \\
\text { psicossocial associado ao desempenho } \\
\text { musical, onde a VFC aumentou após o uso } \\
\text { da terapia, evidenciando um aumento da } \\
\text { influência parassimpática na FC. }\end{array}$ \\
\hline $\begin{array}{l}\text { Alvares et al. } \\
2013^{6}\end{array}$ & $\begin{array}{l}\text { SDNN; RMSSD; } \\
\text { HF; LF }\end{array}$ & $\begin{array}{l}\text { Examinar se a VFC foi reduzida } \\
\text { após tratamento de pacientes } \\
\text { diagnosticados com transtorno } \\
\text { de ansiedade social. }\end{array}$ & $\begin{array}{l}53 \text { pacientes tratados } \\
\text { para transtorno de } \\
\text { ansiedade e para o grupo } \\
\text { controle foram escolhidos } \\
53 \text { alunos de uma } \\
\quad \text { universidade. }\end{array}$ & $\begin{array}{l}\text { A ansiedade social está relacionada à uma } \\
\text { VFC reduzida. A VFC pode ser utilizada para } \\
\text { refletir uma inibição comportamental. }\end{array}$ \\
\hline $\begin{array}{l}\text { Yu et al. } \\
2014^{14}\end{array}$ & $\begin{array}{l}\mathrm{LF}\left(\mathrm{ms}^{2}\right) ; \mathrm{HF} \\
\left(\mathrm{ms} \mathrm{s}^{2}\right) ; \mathrm{LF}(\mathrm{nu}) ; \\
\mathrm{HF}(\mathrm{nu}) ; \mathrm{LF} / \mathrm{HF}\end{array}$ & $\begin{array}{l}\text { Investigar os efeitos das } \\
\text { tarefas mentais sobre o } \\
\text { desempenho cardíaco. }\end{array}$ & $\begin{array}{l}54 \text { indivíduos do sexo } \\
\text { masculino, com idade } \\
\text { entre } 22 \text { e } 27 \text { anos. }\end{array}$ & $\begin{array}{l}\text { A atividade do SNA afeta a FC e esta pode } \\
\text { ser diminuída por tarefas de indução mental, } \\
\text { ao diminuir a atividade simpática e aumentar a } \\
\text { atividade parassimpática. }\end{array}$ \\
\hline $\begin{array}{l}\text { Chen et al. } \\
2012^{10}\end{array}$ & HF & $\begin{array}{l}\text { Elucidar a relação entre } \\
\text { depressão e ansiedade com } \\
\text { a função autonômica cardíaca } \\
\text { em idosos. }\end{array}$ & $\begin{array}{l}354 \text { homens com idade } \\
\text { igual ou superior à } 65 \text { anos. }\end{array}$ & $\begin{array}{l}\text { A depressão e a ansiedade tanto em conjunto } \\
\text { como de forma independente exercem } \\
\text { influência sobre o controle vagal cardíaco. }\end{array}$ \\
\hline $\begin{array}{l}\text { Kemp et al. } \\
2012^{12}\end{array}$ & $\begin{array}{l}\text { SDNN; RMSSD; } \\
\text { HF; LF/HF }\end{array}$ & $\begin{array}{l}\text { Determinar em pacientes } \\
\text { saudáveis se a redução da VFC } \\
\text { está relacionada ao transtorno } \\
\text { depressivo maior (MDD), } \\
\text { ansiedade ou distúrbios do } \\
\text { pânico e estresse. }\end{array}$ & $\begin{array}{l}73 \text { pacientes } \\
\text { diagnosticados com MDD } \\
\text { e } 94 \text { indivíduos saudáveis } \\
\text { para o grupo controle. }\end{array}$ & $\begin{array}{l}\text { A VFC foi reduzida nos portadores de MDD em } \\
\text { relação ao grupo controle. Os pacientes que } \\
\text { apresentaram maior nível de ansiedade tiveram } \\
\text { uma redução maior da VFC. }\end{array}$ \\
\hline
\end{tabular}

Siglas/ índices (VFC): SDNN: Desvio padrão de todos os intervalos R-R normais gravados em um intervalo de tempo, expresso em ms; RMSSD: Raiz quadrada da média do quadrado das diferenças entre intervalos R-R normais adjacentes, em um intervalo de tempo, expresso em ms; HF: Componente de alta frequência com variação de 0,15 a 0,4 Hz; LF: Componente de baixa frequência com variação entre 0,04 e 0,15 Hz; LF/HF: Relação entre os componentes de baixa e alta frequência.

Tabela 2: Síntese dos artigos selecionados para a revisão integrativa na segunda busca

\begin{tabular}{|c|c|c|c|c|}
\hline Autor e ano & $\begin{array}{l}\text { Índices } \\
\text { analisados }\end{array}$ & Objetivos & Amostra & Conclusões \\
\hline $\begin{array}{l}\text { Chalmers et al. } \\
2016^{7}\end{array}$ & HF & $\begin{array}{l}\text { Investigar a relação dos } \\
\text { transtornos de ansiedade, } \\
\text { estresse, preocupação e } \\
\text { depressão com a VFC do } \\
\text { estado de repouso. }\end{array}$ & $\begin{array}{l}91 \text { participantes com } \\
\text { idade entre } 17 \text { e } 29 \text { anos. }\end{array}$ & $\begin{array}{l}\text { O sintoma dimensional de preocupação pode } \\
\text { realmente estar conduzindo as reduções de } \\
\text { VFC observadas, pelo menos nos transtornos } \\
\text { de ansiedade. A VFC encontra-se reduzida em } \\
\text { indivíduos com transtornos de ansiedade. }\end{array}$ \\
\hline $\begin{array}{l}\text { Griffiths et al. } \\
2017^{15}\end{array}$ & $\begin{array}{l}\text { FC; RMSSD; HF; } \\
\text { LF; LF/HF. }\end{array}$ & $\begin{array}{l}\text { Analisar a atividade autonômica } \\
\text { em adolescentes com Transtorno } \\
\text { do Déficit de Atenção com } \\
\text { Hiperatividade (TDAH). }\end{array}$ & $\begin{array}{l}229 \text { crianças e } \\
\text { adolescentes com } \\
\text { diagnóstico primário de } \\
\text { TDAH do sexo masculino. }\end{array}$ & $\begin{array}{c}\text { Menor rMSSD e HFA foram associados } \\
\text { com maior ansiedade, comportamentos de } \\
\text { oposição e problemas sociais; a VFC reduzida } \\
\text { foi associada a características comumente } \\
\text { observadas no TDAH e ansiedade. }\end{array}$ \\
\hline $\begin{array}{l}\text { Suh et al. } \\
2013^{9}\end{array}$ & SDNN; HF. & $\begin{array}{l}\text { Investigar as respostas da VFC } \\
\text { à um estressor psicossocial } \\
\text { em pacientes com Doença } \\
\text { Pulmonar Obstrutiva Crônica } \\
\text { (DPOC) e o potencial papel } \\
\text { da ansiedade como fator de } \\
\text { confusão neste relacionamento. }\end{array}$ & $\begin{array}{l}60 \text { indivíduos que } \\
\text { participam do programa } \\
\text { de reabilitação pulmonar } \\
\text { da Universidade } \\
\text { Estadual de Ohio (OSU) }\end{array}$ & $\begin{array}{l}\text { O efeito combinado de ansiedade e DPOC } \\
\text { não teve efeito negativo cumulativo na função } \\
\text { autonômica, contrariamente à hipótese } \\
\text { original, a ansiedade pode desempenhar } \\
\text { um papel mediador nos padrões de VFC em } \\
\text { resposta a um estressor. }\end{array}$ \\
\hline $\begin{array}{l}\text { Sanchez- } \\
\text { Gonzalez } \\
\text { et al. } 2015^{13}\end{array}$ & LF; HF. & $\begin{array}{l}\text { Compreender a associação } \\
\text { entre ansiedade e mudanças } \\
\text { relacionadas à idade na } \\
\text { hemodinâmica, modulação } \\
\text { autonômica e rigidez arterial. }\end{array}$ & $\begin{array}{c}65 \text { adultos } \\
\text { aparentemente } \\
\text { saudáveis: } 43 \text { com idade } \\
\text { entre } 20 \text { a } 29 \text { anos e } 22 \\
\text { com idade entre } 50 \text { e } 60 .\end{array}$ & $\begin{array}{c}\text { A relação entre índices de modulação } \\
\text { autonômica e ansiedade parece ser mediada } \\
\text { através da atividade simpática sustentada e o } \\
\text { controle vagal cardíaco reduzido. Sendo assim } \\
\text { qualquer intervenção que venha a reduzir } \\
\text { a ansiedade pode melhorar a reatividade } \\
\text { cardiovascular ao estresse. }\end{array}$ \\
\hline
\end{tabular}

Siglas/ índices (VFC): SDNN: Desvio padrão de todos os intervalos R-R normais gravados em um intervalo de tempo, expresso em ms; RMSSD: Raiz quadrada da média do quadrado das diferenças entre intervalos R-R normais adjacentes, em um intervalo de tempo, expresso em ms; HF: Componente de alta frequência com variação de 0,15 a 0,4 Hz; LF: Componente de baixa frequência com variação entre 0,04 e 0,15 Hz; LF/HF: Relação entre os componentes de baixa e alta frequência. 


\section{DISCUSSÃO}

A ansiedade está relacionada diretamente com uma redução da VFC. Sendo assim, a utilização da mudança da VFC pode ser útil para explicar a redução da capacidade de engajamento social em casos de ansiedade ${ }^{6}$.

Nesta percepção, Chalmers et al. ${ }^{7}$ e Stratford et al. ${ }^{8}$, mostram também que os indivíduos que apresentam um nível mais elevado de ansiedade possuem uma VFC menor. O que reflete numa capacidade menor do organismo se adaptar frente as alterações fisiológicas.

Contudo, Suh et al. ${ }^{9}$, identificaram que a ansiedade não afetou de forma direta o controle do sistema nervoso autônomo sobre o coração em indivíduos com Doença Pulmonar Obstrutiva Crônica (DPOC). Sendo que as mudanças ocorreram em presença da ansiedade apenas na resposta à um estressor. $\mathrm{O}$ fato dos sujeitos serem portadores de DPOC pode ter alterado a influência da ansiedade sobre o controle cardíaco.

Em relação ao controle do sistema nervoso autônomo sobre o coração, Chen et al..$^{10}$, evidenciaram que a ansiedade, tanto associada à depressão como de forma individual, influencia o controle vagal cardíaco desempenhado pelo sistema nervoso parassimpático. $\mathrm{O}$ índice usado nesse estudo para mensurar a VFC foi o componente de alta frequência (HF), este sendo um indicador da atuação do nervo vago sobre o coração Vanderlei et al. ${ }^{3}$. Porém, a influência do componente simpático não foi analisada.

Nessa mesma concepção, Wells et al. ${ }^{11}$, analisaram os índices simpáticos e parassimpáticos, relatando que níveis aumentados do índice HF e reduzidos da relação LF/HF em pacientes que receberam tratamento para ansiedade expressam preponderância parassimpática sobre a frequência cardíaca quando estão submetidos às situações de estresse. O que sugere um enfraquecimento do controle vagal em presença da ansiedade.

Em contraste, Kemp et al. ${ }^{12}$, relatam um aumento do marcador LF/HF que representa predomínio simpático em detrimento do parassimpático em indivíduos portadores de transtornos de ansiedade, o que foi constatado pelo grupo controle (não ansiosos). Corroborando com isso, Sanchez-Gonzalez et al. ${ }^{13}$, afirmam que a presença da ansiedade provoca uma diminuição dos tônus vagais, aumentando a atividade do sistema nervoso simpático e atenuando a reatividade cardíaca ao estresse.

Nesse sentido, o cérebro interage com o coração e regula a atividade cardíaca em resposta aos estímulos psicológicos. A ansiedade e o estresse são exemplos desses estímulos. O uso de atividades mentais pode aumentar a capacidade cardiológica, Yu et al. ${ }^{14}$. Um aumento elevado da ansiedade durante situações de estresse pode sugerir a vulnerabilidade dos indivíduos às doenças cardiovasculares ${ }^{8}$.

Sanchez-Gonzalez et al. ${ }^{13}$, demostraram que a ansiedade está relacionada à um envelhecimento cardiovascular, mostrando que indivíduos jovens com altos níveis de ansiedade apresentam índices de desempenho cardíaco semelhantes à indivíduos de meia idade com taxas menores de ansiedade. Esses indivíduos jovens ansiosos apresentaram, ainda, uma maior reatividade cardiovascular em comparação com os de sua mesma idade, que possuem uma ansiedade menor.

Além disso, a ansiedade potencializa os efeitos negativos da depressão sobre a VFC, sendo que esses indivíduos que apresentam depressão e ansiedade denotam uma redução maior na VFC comparado aos que possuem apenas os sintomas da depressão ${ }^{12}$.

Esses efeitos podem ser muitas vezes confundidos, uma vez que os transtornos de ansiedade e depressão são muito próximos e semelhantes. O diagnóstico equivocado do transtorno psicológico pode trazer informações erradas sobre suas influências no sistema cardiovascular. É importante que haja essa diferenciação desses transtornos ${ }^{10}$.

A capacidade de participação e comunicação social está relacionada ao controle dos nervos vagos sobre o desempenho cardíaco, os índices que representam a influência desse domínio parassimpático encontram-se reduzidos na presença da ansiedade. Esse fator determina uma menor interação dos portadores de ansiedade com as situações de estresse social ${ }^{15}$.

Em analogia, Alvares et al. ${ }^{6}$, alegam que a ansiedade social está intimamente relacionada com a atividade cardiológica. As medidas que se referem à VFC encontram-se reduzidas nos sujeitos que apresentam esse quadro de ansiedade, possuindo uma menor capacidade de interagir no meio social.

Sugerindo uma alternativa para essa inibição social, Wells et al. ${ }^{11}$, indagam que uma terapia de controle da interação autonômica sobre o coração pode ajudar na excitação fisiológica, propondo que uma maior interação parassimpática com os músculos cardíacos ajuda na redução da ansiedade.

Destaca-se ainda, que vários fatores biológicos podem alterar as medidas da VFC, sendo idade e gênero os principais. Essas variáveis podem confundir a interação da ansiedade com o controle autonômico cardíaco ${ }^{9,13}$.

A ansiedade contribui para o desenvolvimento de um estado de tensão e provoca mudanças nos índices da VFC comparada a de um indivíduo saudável e que não está na condição de ansiedade, condicionando este a um estado de alerta, e assim, ocasiona a prevalência da condução simpática.

\section{CONCLUSÃO}

Verifica-se que a ansiedade exerce influência significativa sobre os marcadores da VFC, sendo essa relação usada na mensuração da atuação do sistema nervoso autônomo sobre o coração. $\mathrm{Na}$ presença da ansiedade a VFC apresenta-se reduzida, o que influi em uma menor adaptação do nosso organismo frente às alterações fisiológicas e ambientais. Essa falta de adaptação pode ser compreendida como uma condição patológica, que reduz a capacidade cardíaca. 


\section{REFERÊNCIAS}

1. Medeiros LA, Ramiro FMS, Lima CAA, Souza LMA, Fortes TMV, Groppo FC. Avaliação do grau de ansiedade dos pacientes antes de cirurgias orais menores. Rev Odontol UNESP. 2013;42(5):357-63. http://dx.doi.org/10.1590/S1807-25772013000500007

2. Lantyer AS, Varanda CC, Souza FG, Padovani RC, Viana MB. Ansiedade e qualidade de vida entre estudantes universitários ingressantes: avaliação e intervenção. Rev Bras Terapia Comp Cog. 2016;18(2):4-19.

3. Vanderlei LCM, Pastre CM, Hoshi RA, Carvalho TD, Godoy MF. Noções básicas de variabilidade da freqüência cardíaca e sua aplicabilidade clínica. Rev Bras Cir Cardiovasc.2009;24(2):205-17. http://dx.doi.org/10.1590/S0102-76382009000200018

4. Santana MDR, Souza ACA, Abreu LC, Valenti VE. Association between oral variables and heart rate variability. Int Arch Med. 2013;6:49. http://dx.doi.org/10.1186/1755-7682-6-49

5. Silva JDA, Padovani RC, Viana MB. O emprego do Biofeedback como Estratégia de Manejo do Estresse e da Ansiedade em Atletas: um Ensaio Clínico. Rev Bras Terapia Comp Cog. 2016;18(3):17-29.

6. Alvares GA, Quintana DS, Kemp AH, Van Zwieten A, Balleine BW, Hickie IB, et al. Reduced heart rate variability in social anxiety disorder: associations with gender and symptom severity. PLoS One. 2013;8(7):e70468

http://dx.doi.org/10.1371/journal.pone.0070468

7. Chalmers JA, Heathers JAJ, Abbott MJ, Kemp AH, Quintana DS. Worry is associated with robust reductions in heart rate variability: a transdiagnostic study of anxiety psychopathology. BMC Psychol. 2016;4:32.

http://dx.doi.org/10.1186/s40359-016-0138-z

8. Stratford T, Meara A, Psychotherapy MG, Lal S. Heart rate variability and the anxious client: cardiac autonomic and behavioral associations with therapeutic alliance. J Nerv Ment Dis. 2014;202(8):613-9. http://dx.doi.org/10.1097/NMD.0000000000000163
9. Suh S, Ellis RJ, Sollers JJ, Thayer JF, Yang HC, Emery CF. The effect of anxiety on heart rate variability, depression, and sleep in chronic obstructive pulmonary disease. J Psychosom Res. 2013;74(5):407-13.

http://dx.doi.org/10.1016/j.jpsychores.2013.02.007

10. Chen HC, Yang CCH, Kuo TBJ, Su TP, Chou P. Cardiac vagal control and theoretical models of co-occurring depression and anxiety: A cross-sectional psychophysiological study of community elderly. BMC Psychiatry. 2012;12:93

https://dx.doi.org/10.1186/1471-244X-12-93

11. Wells R, Outhred T, Heathers JAJ, Quintana DS, Kemp AH. Matter over mind: a randomised-controlled trial of single-session biofeedback training on performance anxiety and heart rate variability in musicians. PLoS One. 2012;7(10):e46597. https://dx.doi.org/10.1371/journal.pone.0046597

12. Kemp AH, Quintana DS, Felmingham KL, Matthews S, Jelinek HF, Hashimoto K. Depression, comorbid anxiety disorders, and heart rate variability in physically healthy, unmedicated patients: implications for cardiovascular risk. PLoS One. 2012;7(2):e30777. https://dx.doi.org/10.1371/journal.pone.0030777

13. Sanchez-Gonzalez MA, Guzik P, May RW, Koutnik AP, Hughes $\mathrm{R}$, Muniz S, et al. Trait anxiety mimics age-related cardiovascular autonomic modulation in young adults. J Hum Hypertens. 2015;29(4):274-80.

https://dx.doi.org/10.1038/jhh.2014.72

14. Yu X, Zhang C, Zhang J. Causal interactions between the cerebral cortex and the autonomic nervous system. Sci China Life Sci. 2014;57(5):532-38.

https://dx.doi.org/10.1007/s11427-014-4627-0

15. Griffiths KR, Quintana DS, Hermens DF, Spooner C, Tsang TW, Clarke $\mathrm{S}$, et al. Sustained Attention and Heart Rate Variability in children and adolescents with ADHD. Biol Psychol. 2017;124;11-20. https://dx.doi.org/10.1016/j.biopsycho.2017.01.004 